\title{
Human-centred Product Architecture from UPPA to SAPAD
}

\author{
Fei $\mathrm{Hu}^{1, *}$, Xi Zhang ${ }^{1}$, Keiichi Sato ${ }^{2}$, S.Teeravarunyou ${ }^{2}$ and Hao Lin $^{1}$ \\ ${ }^{1}$ School of Arts Design, Guangdong University of Technology, No.729, Dongfengdong Road, Guangzhou City, Guangdong \\ Province, China \\ ${ }^{2}$ Institute of Design, Illinois Institute of Technology, 350 N. LaSalle Street, Chicago, Illinois 60610 USA \\ *Corresponding author
}

\begin{abstract}
The proposed of PA (product architecture) makes the product development varied and effective. However, in the current society, focusing on the product to develop new products isn't satisfying the user's needs that become abundant and uneasy to be found. PA provides a systematic research framework that structures the new product from the object (component, unit, object) to object, which is physical architecture logic. The research method UPPA proposed user process and user function, which considers more human factors and adds it into the research process. What's more, based on UPPA, SAPAD constructs the signification transformation mechanism from object (component, unit, object), behavior (user process) and signification to product, in order to excavate the core requirement of human.
\end{abstract}

Keywords-component; human-centred; PA; UPPA; SAPAD

\section{INTRODUCTION}

In recent years, the uncertainty of PA (Product Architecture) is increasingly concerned. Moullec and Jankovic (2012) proposed applying Bayesian nets method to PA. Geddawy and Maraghy (2013) discussed the granularity standard in modular product architecture, applied cladistics method of DSM (design structure matrix) to reconstruction, and proposed hierarchical clustering. Ko (2013) used Boolean matrix, a kind of efficient and flexible fuzzy design structure matrix, to increase the diversity of products and reduce the amount of components iteration.

These researches are widespread and far-reaching, but it conducts PA focused on the dimension of object. At the same time, human-centered design has been widely recognized in the design field. From the perspective of human, PA is firstly used to construct product based on consumer requirement by $\mathrm{Yu}$, Zugasti and Otto (1999). Teeravarun and Sato (2002) introduced the concept of "process" on the basis of PA and proposed "Use Process Based Product Architecture" (UPPA). The process of using product framework by user can most reflect the product structure of user's needs. Meanwhile, based on UPPA, Hu and Sato (2012) put forward a new framework 'Semiotics Approach of Product Architecture Design' (SAPAD) to analyze the mapping between behavior, signification and product, and explore product architecture from the perspective of signification.

\section{ON UPPA}

\section{A. Introduction}

UPPA (Use Process Based Product Architecture) is a concept brought in process of product development, which includes function, process and product. The process and product are interdependence. The process can't be described without product. The process can show product structure that is best to embody user need. Figure I describes the relationship between three elements of model and obtaining the process by product structure. It is called user function that relate to functional elements.

Using process architecture is mapped to product architecture with UPPA. It is the result that idea of producer is consistent with that of user. Product architecture connects to using process architecture by user function. User function is not an inherent part of product structure, so user function need keep consistent with system function. User function regards as a bridge between using process architecture and product architecture.

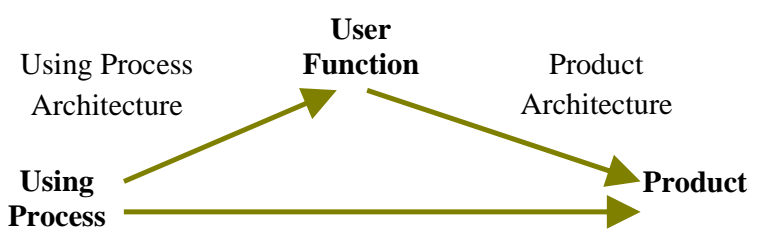

FIGURE I. THE ARCHITECTURE OF USER PROCESS BASED PRODCUT ARCHITECTURE.

\section{B. The Research Process of UPPA}

As figure II shows, user process based product architecture is an interactive process.

- (1) The collection of user data is the beginning of construction of use process.

- (2) Decompose the use process into subtask.

- (3) Once the user tasks create based on user knowledge or user data, it is brought into the module of use process. In this way can complete the construction of use process. 
- (4) User function is an intermediary between the construction of user process and product construction. There are three steps of product construction.

- (5) Create additional functions to complete the function structure.

- (6) Group functions into several groups and create behavior structure.

- (7) Behavior components are grouped by final form.

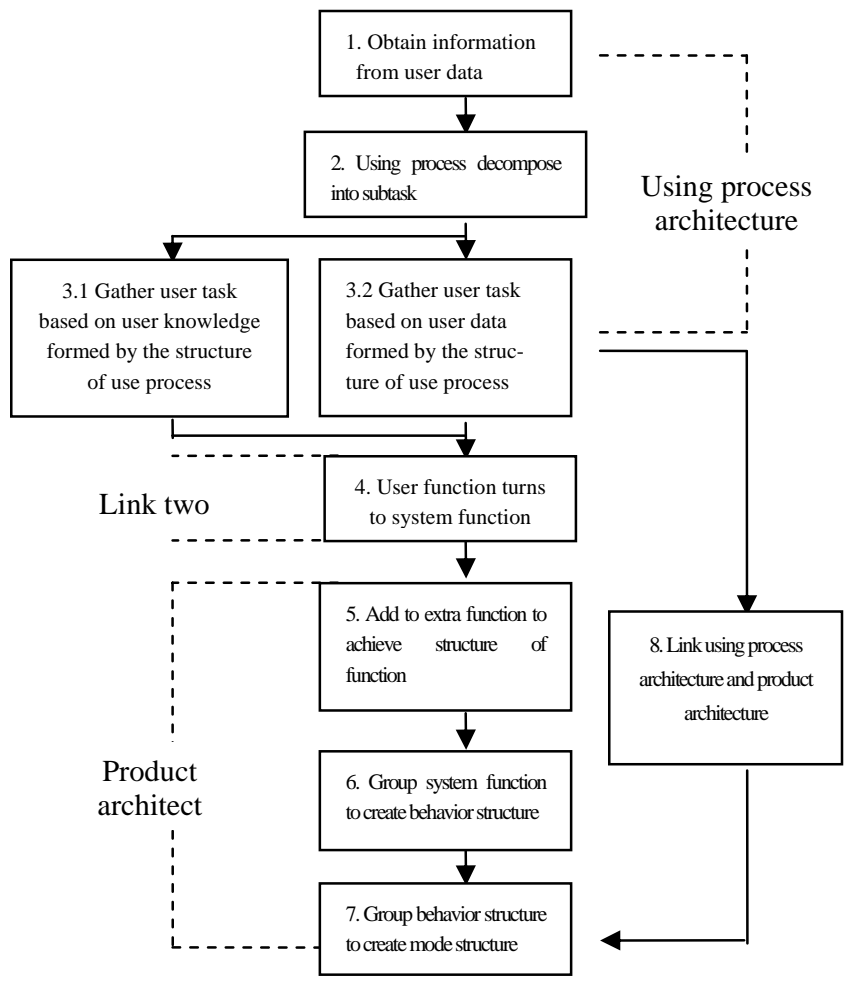

FIGURE II. THE PROCESS OF UPPA.

\section{ON SAPAD}

UPPA introduces the process of product using from the users prospective and named it 'user process' that is focus on the user experience rather than the function of product itself. The really using process is presented by user process, which is the effective and specific method to find the user function. Therefore, the mapping process from user experience to user function is achieved. In the end, the product architecture will be built based on the Function-Behavior-Physical Structure. It is important to get the user experience from the user process when applied the UPPA.

However, user process needs to be explained by people when applied the UPPA method to find the user experience, which chooses a certain process module based on the designer's reason. Therefore, it is need to establish the transformation mechanism between structure of user process and product structure to solve the problem that it need people to explain, in order to reduce time and keep the consistency.
Based the UPPA method, the research method of SAPAD also takes the user process as the starting and foundation of research. Meanwhile, the SAPAD provides a systematized research framework for the explanation of user experience in the user process. The SAPAD introduces the concept of Semiotic Ladder by Stamper and makes it to develop into a signification system framework for explaining the user behavior and object, which will make the researcher to excavate the deep and varied signification, such as the user experience, emotion, knowledge, culture. Signification becomes the transformation mechanism between the user's requirement and product architecture.

\section{A. Introduction}

Generally, human-computer/product interaction (HCI) includes two dimensions: subjective dimension (user's behavior) and objective dimension (feedback of product). The author regards the HCI system as a semiotic system in which behavior act as "Media", product act as "Object", signification act as "Interpretation", referring to the Peirce's trichotomy. Therefore, acting as "Interpretation" for motivation and demands, signification becomes the third dimension. Referring to the Peirce's research continually the conception of hierarchy was introduced into signification-behavior-object. Referring to the theory of Product Architecture, the dimension of object can be divided into four levels: assembly, object, unit and component. Component includes the activity of all products that was applied for completing task in the process. Every product can be divided into some parts based on different aims and every unit is composed of many components. Referring to the behavior science, the dimension of behavior can be divided into four levels: activity, process, action and operation.

Finally, In the dimension of signification, compared the Morris' semiotic triangle theory (1938), Nauta's semiotic cube theory, Stamper's semiotic ladder theory and Cordeiro and Filipe's semiotic pentagram theory, the author finally define the level of human computer/product interaction which is base on Stamper's semiotic ladder theory. It includes six levels as follow:1) Physical level of signification is about "what" in physical attribute, which is related to function, such as material, signals, traces and physical distinctions; 2) Syntactic level of signification is about "how" to connect with each other between the function modules; 3) Empiric level of signification is about "how" to connect the subject with object between the interaction and it relates to construction of logic, which focus on the operation and control of object, the users' experience such as mode, way, noise, redundancy and efficiency etc.; 4) Semantic level of signification is about "why" to interact between individual and object, which relate to emotional experience and focus on emotion, character and persuasiveness of object, such as theme, expression, and intention; 5) Pragmatic level of signification is about "how" to communicate in interactions, which focuses on sub-culture and group identity; 6) Social level of signification is about social attributes in the interaction, which focuses on value and ideology and relates to beliefs, expectation, commitment, contract, law and cultural convention. The three dimensions of SAPD framework are shown in Figure III. 


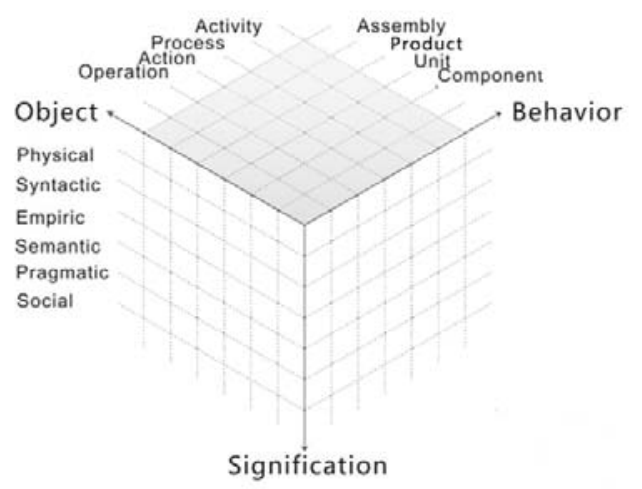

FIGURE III. THE THREE DIMENSIONS OF SAPAD FRAMEWORK.

\section{B. The Research Process of SAPAD}

First part: Behavior observation and analysis. It includes 3 steps: 1) Object analysis. Using PA method to analyze objects (product or service system) and create product schematic, cluster schematic elements, establishing a rough geometric layout, determining basic and occasional interaction (Ulrich, 1995). 2) Users observation. Applying ethnographic method mainly torecord the relation of user behavior, environment and objects by videoing, photographing, noting and other forms. 3) Behavior analysis. Applying the Analytic Hierarchy Process to make behavior hierarchy and layer behavior according to activities, processes, action and operation, outlining the structure of the user's behavior and related items clearly.

Second part: Signification analysis and construction. It includes 2 steps: 4) Excavating the undermeaning of the user's behavior by analyzing physical level, syntactic level, experience level, semantic level, pragmatic level and social level. 5) Signification construction. Making sure of the accuracy and availability of signification through interviewing user again, at the same time, reconstructing signification cluster, insight into the crucial meaning of behavior and core values of the user and possible design directions by matrix hierarchical clustering.

Third part: Product construction and design. It includes 3 steps: 6) Signification-Objects mapping. Combining with 4), $3)$, 2) to determine the mapping among and between signification cluster (four levels) and things, defining the key objects of signification. 7) Product architecture bases on signification cluster. Assigning the number to the relationship of object signification base on $\{0,3\}$ Brin logic and outputting new units, new products or new groups by symmetric matrix. 8) Design opportunities. Defining new function and new structures of product, legible design opportunities and concrete paths for innovation base on the new combination based on component, unit, object and assembly levels. The research flow of SAPAD is shown in Figure IV.

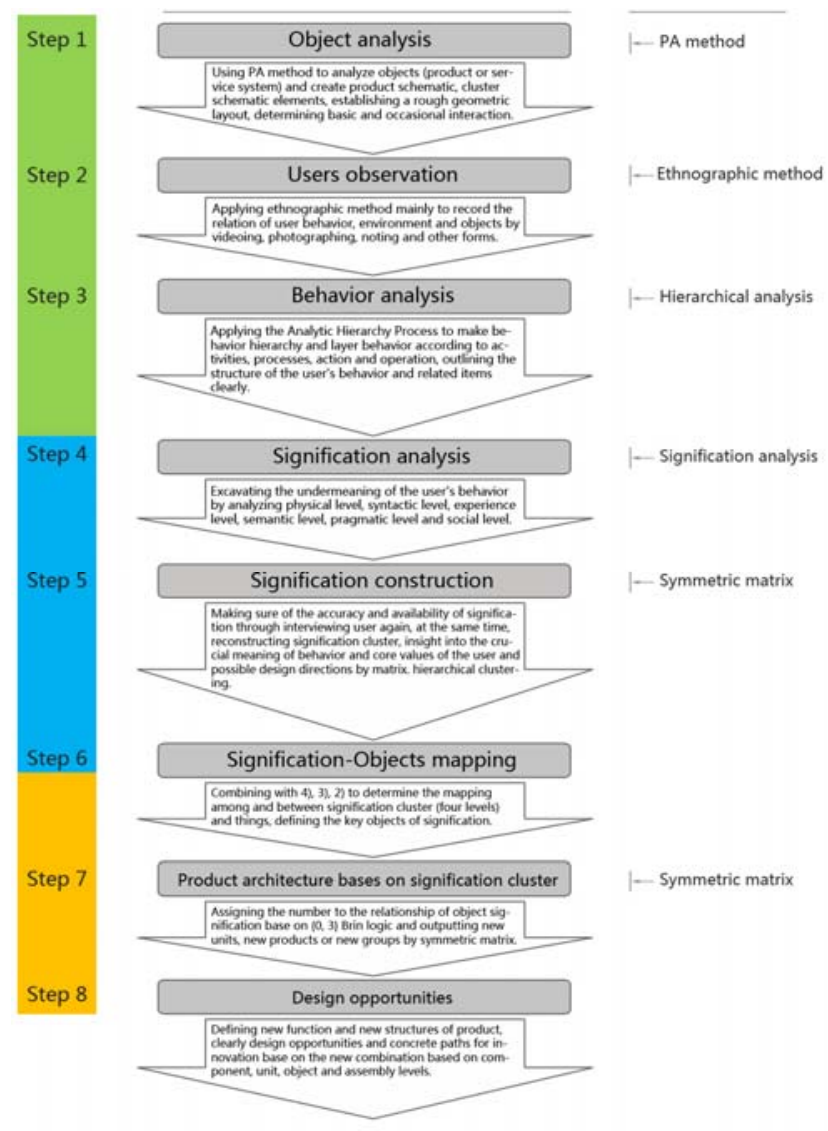

FIGURE IV. THE RESEARCH PROCESS OF SAPAD.

\section{DISCUSSION}

PA (Product Architecture) theory is a manufactureoriented product development, whose essence is product function. The method of modularization is used to allocate product function elements to entity building units of product; from the perspective of semiotics ladder, Product Architecture theory involves the signification system of the Physical level and Syntactic level.

UPPA (Use Process Based Product Architecture) framework adds the concept of "using process" to Product Architecture theory, which opens a user-centered way for the product structure. From the perspective of semiotics ladder, UPPA framework involves the signification system of the physical level, syntactic level and empiric level.

SAPAD introduces the systematic framework of signification analysis after defined the user process. It not only focuses but also excavates the user needs from the behavior and object to signification in order to get the core requirement of user.

\section{CONCLUSIONS}

On modular product architecture, user related issues such as usability and affordance had been addressed enough. However, the rich and colorful meanings such as emotion, community, and culture are still ignored. SAPAD(Semiotic Approach to Product Architecture Design)is to incorporate 
how users embed, develop and interpret meaning and values in locally ordinary living. Signification excavating is not the ultimate goal. In terms of the discovery of functional signification of the physical level and syntactic level, or individual experience and emotion excavated from the experience level and semantic level, or socially cultural signification excavating from pragmatic and social level, the ultimate goal is to translate it into useful design knowledge that can be converted into products by designer.

\section{ACKNOWLEDGMENT}

This study was supported by supported by higher education teaching reform project of Guangdong Province education Department on "T style integrated innovation design knowledge system and enterprise cooperative education mechanism research" (No.JGXM002); supported by the introduction of Guangdong Innovation Research Team Program on "Industrial Design Integrated Innovation Research Team” (No.2011G089); supported by high level talent project of colleges and universities in Guangdong Province "Research on Semiotics Approach to Product Architecture Design”.

\section{REFERENCES}

[1] Sakol. An approach to user knowledge and product architecture for knowledge lifecycle. IIT: [Doctoral Dissertation], 2002.

[2] S. Teeravarunyou and K. Sato, "User Process Based Product Architecture," in The World Congress on Mass Customization and Personalization, Hong Kong, October 1 - 2, pp. 1 - 10.

[3] Stamper, R. (1996) 'Signs, Norms, and Information Systems'. In Holmqvist, B. et al (eds.), Signs at Work, Walter de Gruyter, Berlin, Germany,pp. 349-397.

[4] Ulrich K. (1995) 'The role of product architecture in the manufacturing firm', Research Policy. Vol. 24, no. 3, pp.419-441.

[5] Yu S. J., Gonzalez-Zugasti P. J. and Otto N. K. (1999) 'Product Architecture Definition Based Upon Customer Demands", Journal of Mechanical Design, vol. 129, no. 3, pp. 329-335.

[6] Roland P. (1987) 'Charles Morris and the Behavioral Foundations of Semiotics', Classics of Semiotics. Ed. Krampen Plemun Press. New York: 25.

[7] Morris, C. (1968) Signification and significance: A Study of the Relations of Signs and Values, MIT Press, Cambridge, MA.

[8] Nauta D. (1972) The Meaning of Information, The Hague: Mouton De Gruyter. 